\title{
CONTINUOUS TREE-LIKE SCALES
}

\author{
JAMES CUMMINGS
}

\begin{abstract}
Answering a question raised by Luis Pereira, we show that a continuous tree-like scale can exist above a supercompact cardinal. We also show that the existence of a continuous tree-like scale at $\aleph_{\omega}$ is consistent with Martin's Maximum.
\end{abstract}

\section{INTRODUCTION}

In his $\mathrm{PhD}$ thesis [12] Luis Pereira introduced the notion of a PCF-theoretic object called a tree-like scale, and showed that there is a connection between the existence of such scales and Shelah's PCF Conjecture. Pereira raised the question as to whether a continuous tree-like scale can exist at singular cardinals above a supercompact cardinal. We give a positive answer to this question. Before stating our results more precisely, we give some background.

We recall that if $\left\langle\mu_{n}: n<\omega\right\rangle$ is an increasing sequence of regular cardinals, then a scale of length $\lambda$ in $\prod_{n} \mu_{n}$ (modulo finite sets) is a sequence $\left\langle f_{\alpha}: \alpha<\lambda\right\rangle$ which is increasing and cofinal in $\left(\prod_{n} \mu_{n},<^{*}\right)$, where $f<^{*} g$ if and only if $f(m)<g(m)$ for all large $m$. When $\lambda=\left(\sup _{n} \mu_{n}\right)^{+}$we will call such sequences scales in $\prod_{n} \mu_{n}$. If $\mu$ is a singular cardinal of cofinality $\omega$ then a scale at $\mu$ is a scale in $\prod_{n} \mu_{n}$ for some increasing sequence $\left\langle\mu_{n}: n<\omega\right\rangle$ of regular cardinals cofinal in $\mu$.

If $\beta<\lambda$ is a limit ordinal of uncountable cofinality, then $h$ is an exact upper bound (eub) for $\left\langle f_{\alpha}: \alpha<\beta\right\rangle$ if and only if

$$
\left\{g: g<^{*} h\right\}=\left\{g: \exists \alpha<\beta: g<^{*} f_{\alpha}\right\} .
$$

Such an $h$ may not exist, but if it exists it is unique modulo finite: the scale is continuous if and only if at every $\beta<\lambda$ where an eub for $\left\langle f_{\alpha}: \alpha<\beta\right\rangle$ exists, $f_{\beta}$ is such an eub. The scale is good if and only if for every $\beta<\lambda$ with $c f(\beta)>\omega$ there is an eub $g$ for $\left\langle f_{\alpha}: \alpha<\beta\right\rangle$ such that $c f(g(n))=c f(\beta)$ for all $n$.

The following definition appears as Definition 5.11 in [12].

Definition 1. A scale $\left\langle f_{\alpha}: \alpha<\lambda\right\rangle$ in $\prod_{n} \mu_{n}$ is tree-like if and only if for every $n<\omega$ and every $\alpha<\beta<\lambda$, if $f_{\alpha}(n)=f_{\beta}(n)$ then $f_{\alpha} \uparrow n=f_{\beta} \uparrow n$.

We note that if $\left\langle f_{\alpha}: \alpha<\lambda\right\rangle$ is tree-like, then there are functions $\left\langle F_{n}: \mu_{n+1} \rightarrow\right.$ $\left.\mu_{n}: n<\omega\right\rangle$ such that $F_{n}\left(f_{\alpha}(n+1)\right)=f_{\alpha}(n)$ for all $\alpha, n$.

Pereira [12] proved that the existence of enough continuous tree-like scales can imply cases of Shelah's PCF Conjecture. We will sketch the proof of an interesting special case. Recall that the PCF Conjecture states that for any set $A$ of regular cardinals with $|A|<\min (A),|p c f(A)|=|A|$.

2000 Mathematics Subject Classification. 03E04, 03E55.

Key words and phrases. Supercompact cardinals, tree-like scales, PCF theory, forcing. 
Shelah [14] proved some results which relate the failure of the PCF Conjecture to the existence of free subsets. In particular if the PCF Conjecture fails for the set $\left\{\aleph_{n}: 1<n<\omega\right\}$ then there exist

(1) An infinite set $X \subseteq \omega$,

(2) A regular cardinal $\theta$,

(3) An internally approachable structure $N \prec H_{\theta}$ of length and cardinality $\aleph_{m}$ for some $m<\min (X)$, containing everything relevant,

such that for all $n \in X$

$$
\chi_{N}\left(\aleph_{n}\right) \notin S k\left(N \cup\left\{\chi_{N}\left(\aleph_{m}\right): m \in X, m \neq n\right\}\right) .
$$

We claim that under these circumstances there is no continuous tree-like scale in $\prod_{n} \aleph_{n}$. If there is a continuous tree-like scale in $\prod_{n} \aleph_{n}$ then there is such a scale $\left\langle f_{\alpha}: \alpha<\aleph_{\omega+1}\right\rangle$ lying in $N$, and also there exists in $N$ a sequence of functions $F_{n}$ such that $F_{n}\left(f_{\alpha}(n+1)\right)=f_{\alpha}(n)$ for all $\alpha, n$. Let $\gamma=\sup \left(N \cap \aleph_{\omega+1}\right)$, then a standard argument from PCF theory shows that $f_{\gamma}(n)=\sup \left(N \cap \aleph_{n}\right)$ for all large $n$. But then $F_{n}\left(\sup \left(N \cap \aleph_{n+1}\right)\right)=\sup \left(N \cap \aleph_{n}\right)$ for all large $n$, contradicting (1).

Pereira had conjectured that continuous tree-like scales should exist, and proposed this as a line of attack on the PCF Conjecture. In particular he conjectured that when $\kappa$ is singular strong limit of cofinality $\omega$ and $2^{\kappa}=\lambda>\kappa^{+}$there will always exist a continuous tree-like scale of length $\lambda$. However Gitik [9] refuted this conjecture, producing (among other results) a model where $2^{\kappa}=\kappa^{++}$and there is no continuous tree-like scale of length $\kappa^{++}$.

Pereira also raised the question whether continuous tree-like scales should exist above a supercompact cardinal. The question is quite a natural one, as supercompact cardinals exert a strong influence on the combinatorics of singular cardinals above them: for example

(1) (Solovay) If $\kappa$ is supercompact then the Singular Cardinals Hypothesis holds at singular cardinals above $\kappa$.

(2) (Shelah) If $\kappa$ is supercompact then there are no good scales at singular cardinals of cofinality $\omega$ above $\kappa$.

We note that by work of Shelah a failure of SCH above a cardinal $\kappa$ implies the existence of a good scale above $\kappa$, so that Shelah's result implies Solovay's. It is also interesting to note that there are versions of Shelah's result in which $\kappa$ is a small cardinal with some form of compactness property; for more on this see Foreman and Magidor's paper [8] on very weak squares and Magidor and Shelah's paper [11] on almost-free non-free structures

We finish this introduction by stating the main results of this paper.

Theorem 1: If $\kappa$ is supercompact then there is a generic extension in which $\kappa$ is supercompact and there is a continuous tree-like scale of length $\kappa^{+\omega+1}$ in $\prod_{n} \kappa^{+n}$. Theorem 2: If $\kappa$ is supercompact there is a generic extension in which $\kappa=\omega_{2}$, Martin's Maximum holds and there is a continuous tree-like scale of length $\aleph_{\omega+1}$ in $\prod_{n} \aleph_{n}$.

\section{Continuous tree-Like SCALES ABOve A SUPERCOMPACT CARDinAL}

We will produce by forcing a continuous tree-like scale on $\prod_{n} \kappa^{+n}$ where $\kappa$ is supercompact. The argument can readily be adapted to more general situations. We use a forcing poset defined by Pereira [12, section 5.3].

Given $\alpha$ inaccessible let $\mathbb{Q}(\alpha)$ be the following two step iteration: 
(1) $\mathbb{Q}^{0}(\alpha)$ is the full support iteration of length $\omega$ in which we add for each $n<\omega$ a function $F_{n}: \alpha^{+n+1} \rightarrow \alpha^{+n}$ using partial functions of size $\alpha^{+n}$.

(2) $\mathbb{Q}^{1}(\alpha)$ is defined in the extension by $\mathbb{Q}^{0}(\alpha)$. Conditions are continuous $<^{*}$-increasing sequences in $\prod_{n} \alpha^{+n}$ of the form $\left\langle g_{\alpha}: \alpha \leq \beta\right\rangle$ such that $F_{n}\left(g_{\alpha}(n+1)\right)=g_{\alpha}(n)$ for $\alpha \leq \beta$ and $n<\omega$. The ordering is end-extension.

Pereira [12] proved that assuming GCH this forcing poset is $\alpha$-strategically closed, collapses no cardinals and adds a continuous tree-like scale.

Assume that $\kappa$ is supercompact. By a suitable preparatory forcing we may assume that GCH holds. Let $F$ be a Laver function [10] for $\kappa$, that is to say a function $F: \kappa \rightarrow V_{\kappa}$ such that for all sets $x$ and cardinals $\lambda$ there is $j: V \rightarrow M$ with $\kappa=\operatorname{crit}(j), j(F)(\kappa)=x, j(\kappa)>\lambda$, and ${ }^{\lambda} M \subseteq M$.

We will say that a cardinal $\alpha$ is "acceptable" if $\alpha$ is inaccessible and for every $\beta<\alpha$, if $F(\beta)$ is an ordinal then $F(\beta)<\alpha$. We iterate with Easton support for $\kappa+1$ steps, forcing with $\mathbb{Q}(\alpha)^{V^{\mathbb{P}} \alpha}$ at every acceptable $\alpha<\kappa$ together with $\alpha=\kappa$.

We claim that this iteration preserves the supercompactness of $\kappa$. We use essentially the argument of Laver's indestructibility theorem [10], except that we have to work harder for the master conditions because our forcing posets are only $\alpha$-strategically closed.

Let $\lambda>\kappa^{+\omega}$ be a cardinal, let $\mu$ be much larger than $\lambda$ and let $j: V \rightarrow M$ be such that $\kappa=\operatorname{crit}(j), j(\kappa)>\mu, j(F)(\kappa)=\lambda$, and ${ }^{\mu} M \subseteq M$. Let $G$ be the generic object below $\kappa$, and let $g=g_{0} * g_{1}$ be the generic object at $\kappa$.

By the agreement between $V$ and $M, j\left(\mathbb{P}_{\kappa}\right)$ is in $M$ an iteration which prolongs $\mathbb{P}_{\kappa+1}$. We force over $V[G * g]$ to obtain a generic object $H$ for the iteration between stage $\kappa$ and stage $j(\kappa)$.

Since $j$ " $G \subseteq G * g * H$, we may lift to get $j: V[G] \rightarrow M[G * g * H]$ by defining $j: i_{G}(\dot{\tau}) \mapsto i_{G * g * H}(j(\dot{\tau}))$. Note that the next acceptable ordinal past $\kappa$ on the $M$-side is greater than $\lambda$, and so easily $H$ is generic over $V[G * g]$ for forcing which is $\left(2^{\lambda}\right)^{+}$-strategically-closed. Also $M[G * g * H]$ can compute $j\left\lceil H_{\lambda^{+}}^{V[G]}\right.$, since both $j\left\lceil H_{\mu}^{V}\right.$ and $G * g * H$ are in $M[G * g * H]$.

To further extend the elementary embedding $j$ to the domain $V[G * g]$, we need to choose a generic object $h_{0} * h_{1}$ over $M[G * g * H]$ for $\mathbb{Q}(j(\kappa))$ such that $j$ " $g_{0} * g_{1} \subseteq$ $h_{0} * h_{1}$. We will do this by constructing in $M[G * g * H]$ a (strong) master condition, that is a condition in $\mathbb{Q}(j(\kappa))$ which is a lower bound for $j$ " $g_{0} * g_{1}$; forcing below this condition will give us $h_{0} * h_{1}$ as required. We present the construction in two steps: we construct a master condition for $g_{0}$, lift the embedding $j$ to the domain $V\left[G * g_{0}\right]$, and then find a master condition for $g_{1}$.

We need to choose a master condition $p_{0}$ for $g_{0}$ with some care, so as to be able to choose a master condition for $g_{1}$. We will define $p_{0}$ coordinate by coordinate, working in the model $M[G * g * H]$. Let $\mu_{n}=\sup \left(j^{\prime \prime} \kappa^{+n}\right)$, and note that $\mu_{n}<$ $j\left(\kappa^{+n}\right)$. We maintain the induction hypotheses:

(1) $p_{0}\lceil i$ is a strong master condition for $j: V[G] \rightarrow M[G * g * H]$ and $g_{0}\left\lceil i\right.$, that is $p_{0} \uparrow i \leq j(q)$ for all $q \in g_{0}\lceil i$. As usual this implies that if $p_{0}\left\lceil i \in h_{i}\right.$ with $h_{i}$ generic over $M[G * g * H]$ we can lift to get $j: V[G]\left[g_{0}\lceil i] \rightarrow M[G * g * H]\left[h_{i}\right]\right.$.

(2) $p_{0} \uparrow i$ forces that the domain of $p_{0}(i)$ is $\mu_{i+1}+1$.

To define $p_{0}(0)$ compute the union of $j\left(F_{0}\lceil\alpha)\right.$ for $\alpha<\kappa^{+}$; this is a partial function from $\mu_{1}$ to $\kappa$, we define $p_{0}(0)$ to extend this function, have domain $\mu_{1}+1$ 
and map $\mu_{1}$ to $\mu_{0}=\kappa$. In general to define $p_{0}(i)$ we force below $p_{0} \uparrow i$ to obtain $h_{i}$ with $j^{\text {" }}\left(g_{0}\lceil i) \subseteq h_{i}\right.$, extend the embedding $j$ to an embedding $j: V[G]\left[g_{0} \uparrow\right.$ $i] \rightarrow M[G * g * H]\left[\overline{h_{i}}\right]$, form the union of the functions $j\left(F_{i} \uparrow \alpha\right)$ for $\alpha<\kappa^{+i+1}$, and extend by one point to get a function with domain $\mu_{i+1}+1$ which maps $\mu_{i+1}$ to $\mu_{i}$. Finally we define $p_{0}(i)$ to be (a name for) the resulting function.

To define a strong master condition for $g_{1}$, we work in $M\left[G * g * H * h_{0}\right]$ where $p_{0} \in h_{0}$. We have an embedding $j: V\left[G * g_{0}\right] \rightarrow M\left[G * g * H * h_{0}\right]$, and the construction of $p_{0}$ gives $j\left(F_{n}\right)\left(\mu_{n+1}\right)=\mu_{n}$ for all $n$. Compute the union of $j(q)$ for $q \in g_{1}$; this is a $\mu_{\omega+1}$-sequence of functions, but is not a condition since it is missing a top element.

By considerations of continuity the only possible candidate ( $\bmod$ finite) for a top element is the function $n \mapsto \mu_{n}$, since (as is easily checked) this function is an eub for $\bigcup j$ " $g_{1}$. This function is a valid candidate because $j\left(F_{n}\right)\left(\mu_{n+1}\right)=\mu_{n}$ for all $n$. Extending $\bigcup j$ " $g_{1}$ by adding the function $n \mapsto \mu_{n}$ at $\mu_{\omega+1}$, we obtain a strong master condition $p_{1}$ for $g_{1}$.

Now we can proceed along the same lines as Laver's indestructibility argument[10]. Forcing below $p_{1}$ we get $h_{1}$ with $j " g_{1} \subseteq h_{1}$, so we obtain $j: V[G * g] \rightarrow M[G * g *$ $H * h]$. We compute the set $U$ of $X \in\left(P_{\kappa} \lambda\right)^{V[G * H]}$ such that $j$ " $\lambda \in j(X)$, and use the closure of the forcing which adds $H * h$ to prove that $U \in V[G * g]$. It is routine to check that $U$ will serve as a witness that $\kappa$ is $\lambda$-supercompact in $V[G * g]$. Since $\lambda$ was arbitrary, we have proved that $\kappa$ is fully supercompact in $V[G * g]$.

We have proved

Theorem 1. If $\kappa$ is supercompact then there is a generic extension in which $\kappa$ is supercompact and there is a continuous tree-like scale of length $\kappa^{+\omega+1}$ in $\prod_{n} \kappa^{+n}$.

\section{Continuous tree-Like scales and Martin's Maximum}

The forcing axiom Martin's Maximum (MM) [7] is known to be consistent relative to a supercompact cardinal, and is widely believed to be equiconsistent with a supercompact cardinal. Some evidence that MM is very strong comes from its effects on the combinatorics of singular cardinals. As we mentioned in the Introduction, Shelah showed that there are no good scales at singular cardinals of cofinality $\omega$ above a supercompact cardinal; Menachem Magidor [2] has shown that under MM there are no good scales at any singular cardinal of cofinality $\omega$.

In this section we show that MM is consistent with the existence of a continuous tree-like scale of length $\aleph_{\omega+1}$ in $\prod_{n} \aleph_{n}$. Since the main ideas of the proof are very similar to those of the last section, we will be somewhat sketchy.

We start by recalling the standard construction [7] of a model of MM, starting with a supercompact cardinal $\kappa$ and a Laver function $F: \kappa \rightarrow V_{\kappa}$. We will build an iteration $\mathbb{P}_{\kappa}$ of length $\kappa$, in which semiproper forcing is iterated with revised countable support: at each stage $\alpha$ such that $F(\alpha)$ is a $\mathbb{P}_{\alpha}$-name for a semiproper forcing we let $\mathbb{P}_{\alpha+1}=\mathbb{P}_{\alpha} * \dot{\mathbb{Q}}_{\alpha}$.

Let $G$ be generic for $\mathbb{P}_{\kappa}$. In $V[G]$ we have $2^{\omega}=\kappa=\omega_{2}$. By a result of Shelah [13] the Semi-Proper Forcing Axiom (SPFA) implies MM, so it is enough to check that SPFA holds in $V[G]$. If $\mathbb{Q} \in V[G]$ is semi-proper and $\left\langle D_{i}: i<\omega_{1}\right\rangle$ are dense open sets then we choose an embedding $j: V \rightarrow M$ which witnesses a high degree of supercompactness for $\kappa$, and is such that $j\left(\mathbb{P}_{\kappa}\right)=\mathbb{P}_{\kappa} * \mathbb{Q} * \mathbb{R}$ for some $\mathbb{R}$. We then lift to get $j: V[G] \rightarrow M[G * g * H]$ where $g$ is $\mathbb{Q}$-generic over $V[G]$. We chose $j$ to witness enough supercompactness that $j " g \in M[G * g * H]$, and one can check that 
$j$ "g generates a filter on $j(\mathbb{Q})$ which meets $j\left(D_{i}\right)$ for each $i$. By elementarity there is a filter on $\mathbb{Q}$ in $V[G]$ which meets each $D_{i}$. So MM holds in $V[G]$ as required.

Let $\kappa$ be supercompact and let GCH hold. Let $\mathbb{P}_{\kappa}$ be the iteration we just described and let $G$ be generic for $\mathbb{P}_{\kappa}$. Then in $V[G]$ we have that $2^{\omega}=\omega_{2}$ and GCH holds at all uncountable cardinals. We will force over $V[G]$ with $\mathbb{Q}={ }_{\operatorname{def}} \mathbb{Q}\left(\omega_{2}\right)$, the forcing which we described in the preceding section and which adds a continuous tree-like scale of length $\aleph_{\omega+1}$. It is routine to check that $\mathbb{Q}$ is countably closed (in particular it is semi-proper) and does not collapse cardinals. Let $g$ be $\mathbb{Q}$-generic over $V[G]$.

We claim that MM holds in $V[G * g]$; as before it suffices to check that SPFA holds. Let $\mathbb{R} \in V[G * g]$ be semi-proper, say $\mathbb{R}=i_{g}(\dot{\mathbb{R}})$ where $\dot{\mathbb{R}} \in V[G]$ is a $\mathbb{Q}$-name for a semi-proper forcing poset. Since $\mathbb{Q}$ is semi-proper, it follows that $\mathbb{Q} * \dot{\mathbb{R}}$ is also semi-proper. Let $H$ be $\mathbb{R}$-generic over $V[G * g]$.

We now choose an elementary embedding $j: V \rightarrow M$ such that $j$ witnesses a high degree of supercompactness of $\kappa$ and $j\left(\mathbb{P}_{\kappa}\right)=\mathbb{P}_{\kappa} * \mathbb{Q} * \mathbb{R} * \mathbb{S}$ for some $\mathbb{S}$. By standard arguments we may extend $j$ to get a generic elementary embedding $j: V[G] \rightarrow M[G * g * H * h]$. We now use the method of the preceding section to build $q \in j(\mathbb{Q})$ which is a strong master condition for $j$ and $g$, that is $q$ is a lower bound for $j$ " $q$; forcing below $q$ we obtain $I$ which is $j(\mathbb{Q})$-generic with $j$ " $g \subseteq I$, and can extend again to get $j: V[G * g] \rightarrow M[G * g * H * h * I]$.

Finally we may argue that $j$ " $H \in M[G * g * H * h * I]$, and that $j$ " $H$ generates a filter on $j(\mathbb{R})$ which meets $j(D)$ for every dense $D \subseteq \mathbb{R}$ with $D \in V[G * g]$. Exactly as in the standard consistency proof for MM, we may use this to argue that SPFA (and hence MM) holds in $V[G * g]$.

We have proved

Theorem 2. If $\kappa$ is supercompact there is a generic extension in which $\kappa=\omega_{2}$, Martin's Maximum holds and there is a continuous tree-like scale of length $\aleph_{\omega+1}$ in $\prod_{n} \aleph_{n}$.

\section{Questions AND Remarks}

As far as we know the only known models without tree-like continuous scales are the ones built by Gitik in [9]. These models are built by Prikry forcing and produce singular strong limit cardinals $\kappa$ of cofinality $\omega$ where $2^{\kappa}=\lambda>\kappa^{+}$and there is no tree-like continuous scale of length $\lambda$. This raises the following questions:

(1) If $\kappa$ is singular strong limit of cofinality $\omega$ and $2^{\kappa}=\kappa^{+}$, does there necessarily exist a tree-like continuous scale of length $\kappa^{+}$at $\kappa$ ?

(2) If $\kappa$ is supercompact and $\lambda>\kappa$ is a singular strong limit cardinal of cofinality $\omega$, does there necessarily exist a tree-like continuous scale of length $\lambda^{+}$at $\lambda$ ?

We note that $\mathrm{SCH}$ always holds above a supercompact cardinal, and that doing Prikry forcing above a supercompact cardinal destroys supercompactness in a rather absolute way (no further cardinal-preserving extension can resurrect supercompactness).

We conclude by mentioning some related work: 
(1) In a series of joint papers [3, 4, 5, 6] with Matt Foreman and Menachem Magidor we have analysed in some detail the interactions between large cardinals, forcing axioms, stationary reflection, L-like combinatorial principles such as squares, and PCF-theoretic scales.

(2) For a regular uncountable $\kappa$, Pereira defined a notion of tree-like continuous scale in the set $\kappa^{\kappa}$ under the eventual domination ordering. Donder observed that the existence of such scales follows from the existence of a universal morass, and Brooke-Taylor and Friedman [1] showed that universal morasses are consistent with large cardinal axioms much stronger than supercompactness.

\section{REFERENCES}

[1] A. Brooke-Taylor and S.D. Friedman, Large cardinals and gap-1 morasses. Ann. Pure Appl. Logic 159 (2009), 71-99.

[2] J. Cummings, Notes on singular cardinal combinatorics, Notre Dame J. Formal Logic 46 (2005), 251-282.

[3] J. Cummings, M. Foreman, and M. Magidor, Squares, scales and stationary reflection. J. Math. Log. 1 (2001), 35-98.

[4] J. Cummings, M. Foreman, and M. Magidor, The non-compactness of square. J. Symbolic Logic 68 (2003), 637-643.

[5] J. Cummings, M. Foreman, and M. Magidor, Canonical structure in the universe of set theory. I. Ann. Pure Appl. Logic 129 (2004), 211-243.

[6] J. Cummings, M. Foreman, and M. Magidor, Canonical structure in the universe of set theory. II. Ann. Pure Appl. Logic 142 (2006), 55-75.

[7] M. Foreman, M. Magidor and S. Shelah. Martin's maximum, saturated ideals, and nonregular ultrafilters. I. Ann. of Math. 127 (1988), 1-47.

[8] M. Foreman and M. Magidor, A very weak square principle. J. Symbolic Logic 62 (1997), $175-196$

[9] M. Gitik. On a question of Pereira. Arch. Math. Logic 47 (2008), 53-64.

[10] R. Laver. Making the supercompactness of $\kappa$ indestructible under $\kappa$-directed closed forcing. Israel J. Math. 29 (1978), 385-388.

[11] M. Magidor and S. Shelah. When does almost free imply free? (for groups, transversals etc.), J. Amer. Math. Soc. 7 (1994), 769-830.

[12] L. Pereira. Combinatoire des cardinaux singuliers et structures PCF. Thesis, University of Paris VII, 2007.

[13] S. Shelah, Semiproper forcing axiom implies Martin maximum but not $\mathrm{PFA}^{+}$, J. Symbolic Logic 52 (1987), 360-367.

[14] S. Shelah. PCF and infinite free subsets in an algebra. Arch. Math. Logic 41 (2002), 321-359. 